\title{
BMJ Open Effectiveness of acupuncture for cancer pain: protocol for an umbrella review and meta-analyses of controlled trials
}

\author{
Yihan He, ${ }^{1,2}$ Yihong Liu, ${ }^{1,2}$ Brian H May, ${ }^{1}$ Anthony Lin Zhang, ${ }^{1}$ Haibo Zhang, ${ }^{1,2}$ \\ ChuanJian Lu, ${ }^{1,2}$ Lihong Yang, ${ }^{1,2}$ Xinfeng Guo, ${ }^{1,2}$ Charlie Changli Xue ${ }^{1,2}$
}

To cite: He Y, Liu Y, May BH, et al. Effectiveness of acupuncture for cancer pain: protocol for an umbrella review and meta-analyses of controlled trials. BMJ Open 2017;7:e018494. doi:10.1136/ bmjopen-2017-018494

- Prepublication history and additional material for this paper are available online. To view these files, please visit the journal online (http://dx.doi. org/10.1136/bmjopen-2017018494).

Received 11 July 2017 Revised 9 November 2017 Accepted 10 November 2017

CrossMark

${ }^{1}$ China-Australia International Research Center for Chinese Medicine, RMIT University, Melbourne, Victoria, Australia ${ }^{2}$ Guangdong Provincial Academy of Chinese Medical Sciences, Guangdong Provincial Hospital of Chinese Medicine and The Second Clinical College, Guangzhou University of Chinese Medicine, Guangzhou, Guangdong, China

Correspondence to Professor Xinfeng Guo; drguoxinfeng@163.com and Dr Charlie Changli Xue; charlie.xue@rmit.edu.au

\section{ABSTRACT}

Introduction The National Comprehensive Cancer Network guidelines for adult cancer pain indicate that acupuncture and related therapies may be valuable additions to pharmacological interventions for pain management. Of the systematic reviews related to this topic, some concluded that acupuncture was promising for alleviating cancer pain, while others argued that the evidence was insufficient to support its effectiveness. Methods and analysis This review will consist of three components: (1) synthesis of findings from existing systematic reviews; (2) updated metaanalyses of randomised clinical trials and (3) analyses of results of other types of clinical studies. We will search six English and four Chinese biomedical databases, dissertations and grey literature to identify systematic reviews and primary clinical studies. Two reviewers will screen results of the literature searches independently to identify included reviews and studies. Data from included articles will be abstracted for assessment, analysis and summary. Two assessors will appraise the quality of systematic reviews using Assessment of Multiple Systematic Reviews; assess the randomised controlled trials using the Cochrane Collaboration's risk of bias tool and other types of studies according to the Newcastle-Ottawa Scale. We will use 'summary of evidence' tables to present evidence from existing systematic reviews and meta-analyses. Using the primary clinical studies, we will conduct meta-analysis for each outcome, by grouping studies based on the type of acupuncture, the comparator and the specific type of pain. Sensitivity analyses are planned according to clinical factors, acupuncture method, methodological characteristics and presence of statistical heterogeneity as applicable. For the non-randomised studies, we will tabulate the characteristics, outcome measures and the reported results of each study. Consistencies and inconsistencies in evidence will be investigated and discussed. Finally, we will use the Grading of Recommendations Assessment, Development and Evaluation approach to evaluate the quality of the overall evidence.

Ethics and dissemination There are no ethical considerations associated with this review. The findings will be disseminated in peer-reviewed journals or conference presentations. PROSPERO registration number CRD42017064113.

\section{Strengths and limitations of this study}

- The three components will provide comprehensive, clinically relevant assessments of the effectiveness and safety of acupuncture and related therapies for cancer pain.

- The umbrella review will provide a quality-appraised overview of existing systematic reviews.

- Meta-analyses will provide assessments of the randomised controlled trial evidence for specific acupuncture therapies and types of cancer pain.

- Analyses of non-randomised studies will identify consistencies and inconsistencies in evidence between study types.

- Anticipated limitations include study quality, diversity between studies and difficulties in synthesising and grading evidence from different types of clinical studies.

\section{BACKGROUND}

For people with cancer, pain is a distressing and intractable symptom with a prevalence rate of over $70 \%{ }^{1}$ Two-thirds of patients in the advanced stage of cancer suffered from pain; more than half of patients undergoing anticancer treatment experienced pain and nearly $40 \%$ of patients after curative treatment still complained about pain. Among these patients, $40 \%$ graded their pain as moderate to severe (Numerical Rating Scale (NRS) Score $\geq 5$ ). ${ }^{2}$ As the possibility of early diagnosis and the improvement of curative technologies prolong survival time for patients affected by cancer, ${ }^{3}$ the requirement for alleviation of pain has been highlighted. ${ }^{4}$ Enormous advancements in knowledge of cancer pain and pain management have been achieved. ${ }^{5}{ }^{6}$ Notably, the WHO analgesic ladder offers a systematic approach to pain relief. ${ }^{78}$ However, cancer pain control remains a critical challenge globally, ${ }^{9} 10$ including issues associated with inadequate pain relief, analgesic addiction and the side effects of pharmacological interventions. ${ }^{11-14}$ 
Clinical studies have shown benefits of acupuncture and related therapies for supportive and palliative care in cancer, by diminishing side effects of conventional therapies, relieving cancer-related concomitant symptoms and improving overall quality of life. ${ }^{14-17}$ Acupuncture and acupressure are listed in the National Comprehensive Cancer Network oncology guidelines as integrative interventions for adult cancer pain. ${ }^{18}$ A detailed guideline has been developed for acupuncture application in the management of pain and non-pain indications in patients with cancer. ${ }^{19}$

Traditionally, acupuncture involves the manual insertion of fine needles into specific loci on the body and its associated techniques include the application of pressure without needles (acupressure) and the application of heat (moxibustion). More modern techniques include electrical stimulation of needles (electroacupuncture) and skin areas (transcutaneous electrical nerve stimulation (TENS)), and the application of light lasers instead of needles (laser acupuncture). ${ }^{20}$

An increasing number of randomised controlled trials (RCTs) of acupuncture for cancer pain have been conducted over the years. From the perspective of research design for clinical trials, acupuncture presents a range of challenges. Acupuncture included a variety of methods and techniques which may not be directly comparable. ${ }^{2021}$ It is difficult to blind personnel and participants in RCTs. Sham/placebo acupuncture devices have been developed, but there is some controversy about whether these and other control methods used in acupuncture studies are truly inert. ${ }^{21-23}$ Another issue is whether the manipulation technique(s) used by the acupuncturist and the number of needles inserted impact on analgesia in a manner similar to medication dosage. ${ }^{2124}$ Further, in traditional acupuncture, the intervention is not standardised and can be adjusted according to the syndrome (Zheng) of the individual patient. ${ }^{25} 26$ One approach to addressing the need to assess acupuncture in realistic settings has been the adoption of pragmatic trial designs. ${ }^{27}{ }^{28}$ Furthermore, in studies of cancer pain, there can be considerable variation in the type of cancer and likely causes of the pain. ${ }^{29}$ All these factors present issues when assessing the effects of acupuncture and related therapies in cancer pain and may limit the extent to which meta-analysis approaches are appropriate.

\section{The need for this work}

One review concluded the accumulated evidence suggested that acupuncture could be beneficial for pain and other symptoms in patients with cancer, ${ }^{30}$ but a Cochrane systematic review concluded that evidence was insufficient to determine whether acupuncture was effective for the management of adult cancer pain. The conclusions were limited by small sample sizes and heterogeneity in methodology, cancer populations and acupuncture techniques. $^{21}$

There are a number of systematic reviews related to acupuncture for cancer pain. ${ }^{21}{ }^{31-38}$ One included acupuncture for pain relief along with other outcomes, ${ }^{35}$ while others discussed acupuncture and other Chinese medicine therapies for cancer care. ${ }^{36} 37$ Of the systematic reviews that explicitly evaluated the effectiveness of acupuncture for cancer pain management, some included RCTs for all types of cancer, ${ }^{33} 34$ while some specifically focused on acupuncture for particular conditions. ${ }^{31} 3238$ Some reviews included Chinese database ${ }^{32-37}$ but others limited searches to English language databases. ${ }^{21} 3138$

All these systematic reviews limited their inclusion criteria to RCTs. However, other types of clinical studies have been published ${ }^{39} 40$ and additional RCTs have been published in recent years. ${ }^{41-44}$ Given the variety of acupuncture and related therapies in current use in integrative cancer therapy and the diversity of cancer populations in which acupuncture has been applied, there is value in conducting a review that aims at integrating multiple levels of evidence. Systematic reviews generally focus on RCTs, but when the RCT evidence is not sufficient to address clinical questions, the inclusion of non-randomised studies can provide supporting evidence, may improve the generalisability of the conclusions, and can inform decision-makers of the totality of the available clinical evidence. ${ }^{45}$

This study takes an umbrella review approach ${ }^{46}$ to summarise the results of published reviews of acupuncture and related therapies for cancer pain. In addition, data from all types of clinical trials will be synthesised and analysed to arrive at a comprehensive evaluation of the effectiveness of each of the acupuncture and related interventions for specific categories of cancer pain.

\section{OBJECTIVES}

Umbrella reviews aim to provide synthesised and appraised evidence on a broad topic area for decision-makers in healthcare, including patients, physicians and policy-makers. ${ }^{46}$ The present study: (1) conducts an umbrella review that aims to synthesise the findings of existing systematic reviews and meta-analyses ${ }^{46}$; (2) conducts a systematic review and meta-analyses of randomised clinical trials and (3) summarises results of other types of clinical studies in order to assess the broad body of clinical evidence, inform clinical practice and identify directions for future clinical trials and other research. The following questions will be addressed: (1) Are acupuncture and related therapies effective for relieving pain associated with cancer? (2) Do acupuncture therapies enable reduction in analgesic consumption? (3) Are the effects (if any) related to the specific type or stage of cancer, the cancer therapies, degree of pain, the type of intervention or other variables?

\section{METHODS}

This protocol was developed according the Preferred Reporting Items for Systematic Reviews and Meta-Analyses Protocols statement (online supplementary file 
1) ${ }^{47} 48$ and has been registered on PROSPERO (no. CRD42017064113). The anticipated start date of this study is $01 / 12 / 2017$.

\section{DESIGN}

This study comprises three main components:

1. An umbrella review of systematic reviews and meta-analyses.

2. A systematic review of randomised controlled studies with meta-analyses of outcome data (if possible).

3. Summaries of the results of non-randomised clinical studies on acupuncture and related therapies for cancer pain.

We have performed a scoping literature review of all cancer pain-related publications in the Cochrane library. This preparatory step helped define the inclusion criteria, develop the literature search strategies and determine the data to be extracted from eligible reviews and clinical studies.

\section{ELIGIBILITY CRITERIA \\ Types of studies}

Articles published in English or Chinese including:

1. systematic reviews and/or meta-analyses of the effects of acupuncture and related therapies on pain relating to cancer;

2. RCTs (with or without blinding) including cross-over designs and pragmatic trials;

3. non-randomised studies, including case-control studies, case series studies, cohort studies, concurrently controlled studies, cross-sectional studies, historically controlled studies, time series studies and case studies. $^{45} 49$

\section{Types of participants}

Adult participants with cancer pain, including pain directly caused by the development of cancer and pain related to treatments for cancer. ${ }^{18}$

\section{Types of intervention}

We will consider acupuncture and related therapies regardless of needling techniques and stimulation method, including manual acupuncture, electroacupuncture, ear acupuncture, acupressure, moxibustion, TENS and combinations of these. Treatments in the comparison groups can be sham/placebo acupuncture, pharmacotherapy or no additional intervention to usual care.$^{5051}$

\section{Types of outcome measures}

We will consider systematic reviews and clinical studies that report numerical data on one or more of the following outcomes:

1. Patient-reported pain intensity or pain relief measured using a Visual Analogue Scale, Verbal Rating Scale, NRS, ${ }^{52}$ the McGill Pain Questionnaire, ${ }^{53}$ the Brief Pain Inventory ${ }^{54}$ or other validated outcome measures. ${ }^{55}$
2. Quality of life measured using validated scales, including the European Organisation for Research and Treatment of Cancer Quality of Life Questionnaire, ${ }^{56}$ the General Version of the Functional Assessment of Cancer Therapy ${ }^{57}$ the Edmonton Symptom Assessment System ${ }^{58}$ or other validated scales. ${ }^{59}$

3. Consumption of analgesics including opioids and non-opioids. ${ }^{18}$

4. Frequency of breakthrough pain ${ }^{60}$ and rescue medication use or dosage.

5. Safety of the acupuncture intervention including adverse events and withdrawals for any reason.

\section{SEARCH STRATEGY}

To identify systematic reviews and/or meta-analyses of acupuncture and related therapies for cancer pain, we will search PubMed (1966 to present), Embase (1974 to present) and CINAHL (1982 to present), JBI Database of Systematic Reviews and Implementation Reports (2003 to present), the Cochrane Database of Systematic Reviews (1992 to present) and the PROSPERO register. ${ }^{46}$ Considering that acupuncture is frequently used in China, we will search the following Chinese databases: Chinese Biomedical Literature Database (CBM, 1978 to present), VIP Database for Chinese Technical Periodicals (CQVIP, 1989 to present), China National Knowledge Infrastructure (CNKI, 1994 to present) and Wanfang Data (1998 to present). The search strategy for PubMed/MEDLINE database is shown in online supplementary file 2.

To identify clinical studies, we will search PubMed, Embase, CINAHL and the Chinese databases CBM, CQVIP, CNKI and Wangfang Data. Search terms will generally consist of three groups: (1) clinical condition (cancer, tumour, carcinoma, neoplasm, pain, analgesia, etc); (2) intervention (acupuncture, electroacupuncture, auriculotherapy, acupoint, needle, acupressure, etc) and (3) study type (random, controlled trial, cohort, casecontrol, comparative study, case series, etc), with adjustments for different databases. ${ }^{61}$

In addition, we will search clinical trial registries, dissertations and grey literature. ${ }^{61}$

\section{Screening and selection}

Following the comprehensive searches for systematic reviews and clinical studies, two reviewers (YHH and YHL) will screen all hits independently based on the titles and abstracts. Full texts will be downloaded for further evaluation when necessary. At the next stage, the reviewers will examine the full text articles according to the inclusion criteria. A third reviewer will be consulted to resolve any disagreement by discussion and consensus. ${ }^{62}$

\section{DATA EXTRACTION}

All data extraction will be undertaken by two independent reviewers (YHH and YHL) who will extract information using predesigned forms. 
For systematic reviews, we will extract ${ }^{4663}$ :

1. identification information (publication year, first author);

2. general information (objectives, type of review, target condition, comparator);

3. methodological characteristics (included study type, any restriction on included studies, databases searched and date ranges, methods of data extraction and assessment of study quality, meta-analysis method);

4. participants (inclusion/exclusion criteria, type of cancer, type of pain);

5. interventions (type of acupuncture and related therapy, type of control intervention);

6. included data (primary and secondary outcomes, number of studies, numbers and types of participants, country and setting of studies);

7. synthesis method, summary and conclusions.

For all types of clinical studies, we will extract ${ }^{626465}$ :

1. identification information (publication year, first author);

2. general information (country, study type, setting, number of centres, sample size, study duration);

3. participants (type and/or stage of cancer, age, sex, type of pain, pain intensity before treatment, Chinese medicine syndrome);

4. interventions (type of acupuncture and related therapy, acupuncture point selection, treatment frequency, number of sessions, duration of each session, point stimulation/manipulation method(s));

5. comparator (if there is any, details of the treatment including name, dosage, frequency and course);

6 . outcomes (data and time points for each measurement, type and number of adverse events in each group).

For both systematic reviews and clinical studies, information related to syndrome/pattern of Chinese medicine will be extracted, including therapeutic principles, Chinese medicine syndrome/pattern and related outcomes. ${ }^{66}$

We will try to contact corresponding authors for any missing data or clarification for unclear information. ${ }^{46} 62$

\section{QUALITY ASSESSMENT}

Two reviewers (YHH and YHL) will appraise the quality of the included reviews independently according to the Assessment of Multiple Systematic Reviews (AMSTAR) tool. ${ }^{67} \mathrm{~A}$ systematic review that reports one item in accordance with AMSTAR receives one point with a maximum score of 11 points. A score of 8-11 represents high quality, while 4-7 means medium quality, and a review with a score below 4 is judged as low quality.

The quality of the RCTs will be assessed using the risk of bias tool developed by the Cochrane Collaboration. We will appraise each study in terms of selection bias (random sequence generation and allocation concealment), performance bias (blinding of participants and personnel), detection bias (blinding of outcome assessment) and attrition bias (incomplete outcome data) ${ }^{68}$

We will carry out quality assessment for non-randomised controlled studies according to the Newcastle-Ottawa Scale for assessing the quality of non-randomised studies in meta-analyses. ${ }^{6} 70$

\section{Evidence synthesis for systematic reviews}

We will report a summary of the findings of the systematic reviews and meta-analyses. For each review, this will include: the type of acupuncture and related therapies; syndrome/pattern in Chinese medicine, the type of cancer pain included (related to neoplasm, related to cancer therapy); the number of included clinical studies; numbers of participants; the outcome measures assessed; any meta-analysis results for each outcome, heterogeneity and reviewers' conclusions. 'Summary of evidence' tables will be used to present syntheses of the overall evidence for each type of cancer pain, type of acupuncture intervention, type of control intervention and outcome measure. $^{46}$

\section{Evidence synthesis for RCTs}

We will use data extracted from published RCTs to perform meta-analyses, calculating effect size and 95\% CI by the random-effects model. Heterogeneity among trials will be identified by the $\chi^{2}$ test and reported as $\mathrm{I}^{2}{ }^{71}$ We will assess publication bias using funnel plots and Egger's test for asymmetry when at least 10 trials are available. ${ }^{72}$

When conducting meta-analysis, for each outcome studies will be grouped according to: (1) the type of acupuncture (eg, manual acupuncture, electroacupuncture, ear acupuncture, acupressure, moxibustion and TENS); (2) the comparator (eg, placebo/sham acupuncture, pharmaceutical therapy, usual care only) and (3) the specific type of pain such as breakthrough pain, ${ }^{73}$ cancer-induced bone pain ${ }^{74}$ or aromatase inhibitor-associated arthralgia. ${ }^{75}$

Sensitivity analyses are planned based on clinical factors (cancer type, degree of pain, Chinese medicine syndrome/pattern), acupuncture method (stimulation method, dosage, specific acupuncture points), methodological characteristics (sample size, risk of bias), and presence of statistical heterogeneity as applicable.

\section{Evidence synthesis for non-randomised studies}

First, we will tabulate the characteristics of each of the non-randomised studies, the outcome measures and the reported results. Where possible, studies will be grouped according to study type, type of acupuncture intervention, type of control (if applicable), type of cancer pain and study quality.

We will compare the results of the meta-analyses of the RCTs with the reported results of non-randomised studies when the studies are similar in terms of acupuncture type and type of cancer pain. If the results for the effects of the RCTs and non-randomised studies are consistent and show the same trend, the evidence from 
the non-randomised studies may provide support that an effect is likely to exist or not exist. On the other hand, if the findings from the non-randomised studies are inconsistent with the evidence from the RCTs, we should be more cautious in the interpretation of the RCT evidence. In balancing the two bodies of evidence, we will need to take a number of factors into consideration including the numbers of participants in the studies, the quality of the studies and the clinical relevance of the interventions. In synthesising the results of the different types of clinical studies, we will consider the non-randomised studies as complementary to the RCTs. ${ }^{45}$

We will use the Grading of Recommendations Assessment, Development and Evaluation approach to evaluate the quality of the overall evidence. ${ }^{76}$

\section{CONCLUDING REMARKS}

This review will build on previous assessments of the evidence for acupuncture and related therapies in the management of cancer pain, by synthesising the results of previous systematic reviews, conducting updated meta-analyses of the outcomes of RCTs, summarising evidence from non-randomised clinical studies and synthesising evidence from multiple sources.

In assessing the results of non-randomised studies, a number of issues will need consideration, including the sources of bias in the selection of participants, confounding effects of variability in baseline characteristics, issues relating to potential bias in participant-reported outcomes and the effects of attrition bias. ${ }^{77-80}$

In synthesising and comparing the results of non-randomised studies and RCTs, we will need to pay attention to issues relating to the external validity of the interventions, whether the reported results from these clinical studies may be generalised to clinical populations, and whether the interventions reflect current clinical practice. ${ }^{81-83}$ In cases where no RCT evidence is available for a particular intervention, type of cancer pain or outcome measure, we will need to base assessments on the available non-randomised studies while acknowledging the limitations of this level of evidence. ${ }^{45}$ In such cases, the non-randomised studies may indicate future directions for RCTs, for example, the pilot study on acupuncture for cancer-induced bone pain ${ }^{39}$ and the pragmatic pilot study on acupuncture for uncontrolled cancer pain, ${ }^{40}$ both suggest that further RCTs are warranted.

Syndrome/pattern (Zheng) differentiation is an essential notion in the theory of Chinese medicine that informs the selection and application of therapeutic interventions. ${ }^{84}$ RCTs and systematic reviews have examined the effects of syndrome differentiation in a number of disorders. ${ }^{85}{ }^{86}$ Syndrome distribution among patients with advanced cancer with opioid-related constipation has been determined by a cross-sectional study ${ }^{87}$ and treatment based on syndrome differentiation has been suggested ${ }^{66}$ Where possible, this review will examine effects related to the application of syndrome/pattern differentiation in the include studies and determine any implications for future clinical studies.

A likely limitation with the overall quality of the evidence is inadequate methodological reporting in many articles published in Chinese. Consolidated Standards of Reporting Trials and Standards for Reporting Interventions in Clinical Trials of Acupuncture have not been adopted by many Chinese language journals and information required for assessment of risk of bias may not be available in reports of RCTs. ${ }^{88-91}$ Poor quality of reporting in RCTs on acupuncture for cancer pain in Chinese journals makes it difficult to assess the validity of the results. ${ }^{92}$ These issues will be considered when conducting sensitivity analyses based on methodological quality and when interpreting results. ${ }^{93}$

The proposed review has several strengths. The umbrella review component will provide an overview of the field and identify issues relating to meta-analysis in a condition as diverse as cancer pain and highlight considerations that will need to be taken into account in the systematic review component. A strength of the systematic review component is the inclusion of multiple Chinese and English language databases and grey literature which should ensure a comprehensive search of the literature. A further strength is any meta-analyses will be informed by rigorous methodology as detailed in the Cochrane Handbook for Systematic Reviews of Interventions. ${ }^{94}$ The addition of the component on non-randomised studies will strengthen the overall project by providing an extended coverage of the clinical literature which may provide supporting evidence to complement the RCT literature and fill any clinically relevant gaps.

Anticipated limitations and challenges include issues relating the quality of the available evidence as outlined above, diversity between clinical studies precluding data pooling in meta-analyses, small sample sizes limiting confidence in outcomes, and difficulties in synthesising and grading evidence from different types of clinical studies. Heterogeneity between the included studies is likely to lead to bias in the results; therefore, we will exercise caution in the interpretation of the results and take a critical approach when assessing the overall evidence. ${ }^{95-97}$

Despite of these anticipated limitations, evaluating the effectiveness of acupuncture for cancer pain is of great importance for clinical practice given the challenges of pain alleviation in patient care. ${ }^{1-3}$ We will consider the issues relating to the scope and overlap of systematic reviews included in the umbrella review, ${ }^{98}$ whether their conclusions are affected by the results of the updated meta-analysis, and whether the results reported by the non-randomised studies are consistent or inconsistent with the results of the meta-analyses. By identifying the strengths, weaknesses and any gaps in the available clinical evidence with regard to particular types of acupuncture interventions or particular 
outcomes that have been assessed in clinical studies, the results of this review can inform future clinical research. Furthermore, by conducting a comprehensive evaluation of multiple types of studies, we anticipate identifying promising acupuncture interventions for specific clinical applications in the management of cancer pain.

Contributors This protocol was first conceived by $\mathrm{YH}$, with critical contributions from the other authors. XG and CCX obtained funding for this study. $\mathrm{YH}$ and $\mathrm{YL}$ wrote the first draft of the protocol and submitted the registration on PROSPERO. BHM, ALZ and HZ provided help to design and edited the manuscript. C-JL and $L Y$ revised the manuscript. All authors contributed constructive comments on the manuscript and approved the final paper. YH, XG and CCX are the guarantors for the publication and are fully responsible for the paper.

Funding Guangdong Provincial Academy of Chinese Medical Sciences, China, and RMIT University through the China-Australia International Research Centre for Chinese Medicine as well as PhD scholarship support to the first author provided by the School of Health and Biomedical Sciences, RMIT University.

Competing interests None declared.

Provenance and peer review Not commissioned; externally peer reviewed.

Open Access This is an Open Access article distributed in accordance with the Creative Commons Attribution Non Commercial (CC BY-NC 4.0) license, which permits others to distribute, remix, adapt, build upon this work non-commercially, and license their derivative works on different terms, provided the original work is properly cited and the use is non-commercial. See: http://creativecommons.org/ licenses/by-nc/4.0/

(c) Article author(s) (or their employer(s) unless otherwise stated in the text of the article) 2017. All rights reserved. No commercial use is permitted unless otherwise expressly granted.

\section{REFERENCES}

1. Neufeld NJ, Elnahal SM, Alvarez RH. Cancer pain: a review of epidemiology, clinical quality and value impact. Future Oncol 2017;13:833-41.

2. van den Beuken-van Everdingen $\mathrm{MH}$, Hochstenbach LM, Joosten $\mathrm{EA}$, et al. Update on prevalence of pain in patients with cancer: systematic review and meta-analysis. J Pain Symptom Manage 2016;51:1070-90.

3. Phillips JL, Currow DC. Cancer as a chronic disease. Collegian 2010;17:47-50.

4. Luckett T, Davidson PM, Green A, et al. Assessment and management of adult cancer pain: a systematic review and synthesis of recent qualitative studies aimed at developing insights for managing barriers and optimizing facilitators within a comprehensive framework of patient care. J Pain Symptom Manage 2013;46:229-53.

5. Candido KD, Kusper TM, Knezevic NN. New cancer pain treatment options. Curr Pain Headache Rep 2017;21:12.

6. Schreiber JA. We've come a long way: a review of cancer pain management. Oncol Nurs Forum 2013;40:106-8.

7. Ventafridda V, Tamburini M, Caraceni A, et al. A validation study of the WHO method for cancer pain relief. Cancer 1987;59:850-6.

8. Carlson CL. Effectiveness of the world health organization cancer pain relief guidelines: an integrative review. J Pain Res 2016:9:515-34

9. Kim YC, Ahn JS, Calimag MM, et al. Current practices in cancer pain management in Asia: a survey of patients and physicians across 10 countries. Cancer Med 2015;4:1196-204.

10. Wang CH, Lee SY. Undertreatment of caner pain. Acta Anaesthesiol Taiwan 2015;53:58-61.

11. Simone CB, Vapiwala N, Hampshire MK, et al. Cancer patient attitudes toward analgesic usage and pain intervention. Clin J Pain 2012;28:157-62.

12. Pachman DR, Barton DL, Swetz KM, et al. Troublesome symptoms in cancer survivors: fatigue, insomnia, neuropathy, and pain. J Clin Oncol 2012;30:3687-96.

13. Kwon JH. Overcoming barriers in cancer pain management. J Clin Oncol 2014;32:1727-33.

14. Paley CA, Johnson MI. Acupuncture for the management of cancerrelated pain? Pain Manag 2017;7:345-9.
15. Wu X, Chung VC, Hui EP, et al. Effectiveness of acupuncture and related therapies for palliative care of cancer: overview of systematic reviews. Sci Rep 2015;5:16776.

16. Towler P, Molassiotis A, Brearley SG. What is the evidence for the use of acupuncture as an intervention for symptom management in cancer supportive and palliative care: an integrative overview of reviews. Support Care Cancer 2013;21:2913-23.

17. Johnstone PA. Acupuncture as cancer symptom therapy: what a difference a decade makes. J Acupunct Meridian Stud 2011;4:209-13.

18. NCCN Clinical Practice Guidelines in Oncology (NCCN Guidelines $®$ ) Adult cancer pain Version 2.2017 https://www.nccn.org/ professionals/physician_gls/pdf/pain.pdf. 2017.

19. Filshie J, Hester J. Guidelines for providing acupuncture treatment for cancer patients--a peer-reviewed sample policy document. Acupunct Med 2006;24:172-82.

20. Birch S, Lewith G. Chapter 2 - Acupuncture research: the story so far. Acupuncture research. Edinburgh: Churchill Livingstone, 2008:15-35.

21. Paley CA, Johnson MI, Tashani OA, et al. Acupuncture for cancer pain in adults. Cochrane Database Syst Rev 2015;10:CD007753.

22. Ju L, Wu X, Xu D, et al. [Application of placebo acupuncture in randomized controlled trials in the past 10 years in foreign countries]. Zhongguo Zhen Jiu 2016;36:203-6.

23. Zhang CS, Tan HY, Zhang GS, et al. Placebo devices as effective control methods in acupuncture clinical trials: a systematic review. PLoS One 2015;10:e0140825.

24. Zaslawski CJ, Cobbin D, Lidums E, et al. The impact of site specificity and needle manipulation on changes to pain pressure threshold following manual acupuncture: a controlled study. Complement Ther Med 2003;11:11-21.

25. Bian ZX, Xu H, Lu AP, et al. Insights of Chinese medicine syndrome study: from current status to future prospects. Chin J Integr Med 2014;20:326-31.

26. Fang J, Zheng N, Wang $\mathrm{Y}$, et al. Understanding acupuncture based on ZHENG classification from system perspective. Evid Based Complement Alternat Med 2013;2013:1-10.

27. Lundeberg $T$, Lund I, Sing A, et al. Is placebo acupuncture what it is intended to be? Evid Based Complement Alternat Med 2011;2011:1-5.

28. Witt CM. Clinical research on acupuncture - concepts and guidance on efficacy and effectiveness research. Chin J Integr Med 2011;17:166-72.

29. Leppert W, Zajaczkowska R, Wordliczek J, et al. Pathophysiology and clinical characteristics of pain in most common locations in cancer patients. J Physiol Pharmacol 2016;67:787-99.

30. Lu W, Rosenthal DS. Acupuncture for cancer pain and related symptoms. Curr Pain Headache Rep 2013;17:321.

31. Bae K, Yoo HS, Lamoury G, et al. Acupuncture for aromatase inhibitor-induced arthralgia: a systematic review. Integr Cancer Ther 2015;14:496-502.

32. Chien TJ, Liu CY, Chang YF, et al. Acupuncture for treating aromatase inhibitor-related arthralgia in breast cancer: a systematic review and meta-analysis. J Altern Complement Med 2015;21:251-60.

33. Chiu HY, Hsieh YJ, Tsai PS. Systematic review and meta-analysis of acupuncture to reduce cancer-related pain. Eur J Cancer Care 2017;26:e12457.

34. Hu C, Zhang $\mathrm{H}$, Wu W, et al. Acupuncture for pain management in cancer: a systematic review and meta-analysis. Evid Based Complement Alternat Med 2016;2016:1-13.

35. Lau $\mathrm{CH}$, Wu X, Chung VC, et al. Acupuncture and related therapies for symptom management in palliative cancer care: systematic review and meta-analysis. Medicine 2016;95:e2901.

36. Tao W, Luo X, Cui B, et al. Practice of traditional Chinese medicine for psycho-behavioral intervention improves quality of life in cancer patients: a systematic review and meta-analysis. Oncotarget 2015;6:39725-39.

37. Tao WW, Jiang $\mathrm{H}$, Tao XM, et al. Effects of acupuncture, tuina, tai chi, qigong, and traditional chinese medicine five-element music therapy on symptom management and quality of life for cancer patients: a meta-analysis. J Pain Symptom Manage 2016;51:728-47.

38. Yang GS, Kim HJ, Griffith KA, et al. Interventions for the treatment of aromatase inhibitor-associated arthralgia in breast cancer survivors: a systematic review and meta-analysis. Cancer Nurs 2017;40:E26-E41.

39. Paley CA, Johnson MI. Acupuncture for cancer-induced bone pain: a pilot study. Acupunct Med 2011;29:71-3.

40. Garcia MK, Driver L, Haddad R, et al. Acupuncture for treatment of uncontrolled pain in cancer patients: a pragmatic pilot study. Integr Cancer Ther 2014;13:133-40. 
41. Lam TY, Lu LM, Ling WM, et al. A pilot randomized controlled trial of acupuncture at the Si Guan Xue for cancer pain. BMC Complement Altern Med 2017;17:335

42. Greenlee H, Crew KD, Capodice J, et al. Randomized shamcontrolled pilot trial of weekly electro-acupuncture for the prevention of taxane-induced peripheral neuropathy in women with early stage breast cancer. Breast Cancer Res Treat 2016;156:453-64.

43. Hsiung WT, Chang YC, Yeh ML, et al. Acupressure improves the postoperative comfort of gastric cancer patients: a randomised controlled trial. Complement Ther Med 2015;23:339-46.

44. Yeh CH, Chien LC, Lin WC, et al. Pilot randomized controlled trial of auricular point acupressure to manage symptom clusters of pain, fatigue, and disturbed sleep in breast cancer patients. Cancer Nurs 2016;39:402-10.

45. Schünemann HJ, Tugwell $P$, Reeves BC, et al. Non-randomized studies as a source of complementary, sequential or replacement evidence for randomized controlled trials in systematic reviews on the effects of interventions. Res Synth Methods 2013;4:49-62.

46. Aromataris E, Fernandez R, Godfrey CM, et al. Summarizing systematic reviews: methodological development, conduct and reporting of an umbrella review approach. Int $J$ Evid Based Healthc 2015;13:132-40.

47. Moher D, Shamseer L, Clarke M, et al. Preferred reporting items for systematic review and meta-analysis protocols (PRISMA-P) 2015 statement. Syst Rev 2015;4:1.

48. Shamseer L, Moher D, Clarke M, et al. Preferred reporting items for systematic review and meta-analysis protocols (PRISMA-P) 2015: elaboration and explanation. BMJ 2015;349:g7647.

49. Wells GA, Shea B, Higgins JP, et al. Checklists of methodological issues for review authors to consider when including nonrandomized studies in systematic reviews. Res Synth Methods 2013;4:63-77.

50. Birch S. Clinical research on acupuncture. Part 2. Controlled clinical trials, an overview of their methods. J Altern Complement Med 2004;10:481-98.

51. Sherman K, Linde K, White A. Chapter 7 - Comparing treatment effects of acupuncture and other types of healthcare A2 MacPherson, Hugh. In: Hammerschlag R, Lewith G, Schnyer R, eds. Acupuncture research. Edinburgh: Churchill Livingstone, 2008.

52. Ripamonti Cl, Santini D, Maranzano E, et al. Management of cancer pain: ESMO clinical practice guidelines. Ann Oncol 2012;23(Suppl 7):vii139-vii154.

53. Graham C, Bond SS, Gerkovich MM, et al. Use of the McGill pain questionnaire in the assessment of cancer pain: replicability and consistency. Pain 1980;8:377-87.

54. Daut RL, Cleeland CS, Flanery RC. Development of the wisconsin brief pain questionnaire to assess pain in cancer and other diseases. Pain 1983;17:197-210.

55. Burton AW, Chai T, Smith LS. Cancer pain assessment. Curr Opin Support Palliat Care 2014;8:112-6.

56. Aaronson NK, Ahmedzai S, Bergman B, et al. The european organization for research and treatment of cancer QLQ-C30: a quality-of-life instrument for use in international clinical trials in oncology. J Natl Cancer Inst 1993;85:365-76.

57. Cella DF, Tulsky DS, Gray G, et al. The functional assessment of cancer therapy scale: development and validation of the general measure. J Clin Oncol 1993;11:570-9.

58. Bruera E, Kuehn N, Miller MJ, et al. The Edmonton Symptom Assessment System (ESAS): a simple method for the assessment of palliative care patients. J Palliat Care 1991;7:6-9.

59. Jacobsen PB, Davis K, Cella D. Assessing quality of life in research and clinical practice. Oncology 2002;16(9 Suppl 10):133-9.

60. Mercadante S. Breakthrough pain in cancer patients: prevalence, mechanisms and treatment options. Curr Opin Anaesthesiol 2015;28:559-64.

61. Lefebvre C, Manheimer E, Glanville J. Chapter 6: Searching for studies. In: Higgins JPT, Green S, eds. Cochrane handbook for systematic reviews of interventions: Wiley, 2008

62. Higgins JPT, Deeks JJ. Chapter 7: Selecting studies and collecting data. In: Higgins JPT, Green S, eds. Cochrane handbook for systematic reviews of interventions. Chichester (UK): John Wiley \& Sons, 2008.

63. Moher D, Liberati A, Tetzlaff J, et al. Preferred reporting items for systematic reviews and meta-analyses: the PRISMA statement. BMJ 2009;339:b2535.

64. MacPherson H, Altman DG, Hammerschlag R, et al. Revised STandards for Reporting Interventions in Clinical Trials of Acupuncture (STRICTA): extending the CONSORT statement. J Evid Based Med 2010;3:140-55.

65. von Elm E, Altman DG, Egger M, et al. The Strengthening the Reporting of Observational Studies in Epidemiology (STROBE) statement: guidelines for reporting observational studies. Int J Surg 2014:12:1495-9.

66. Zhong LLD, Zheng G, Da Ge L, et al. Chinese herbal medicine for constipation: zheng-based associations among herbs, formulae, proprietary medicines, and herb-drug interactions. Chin Med 2016;11:28.

67. Shea BJ, Grimshaw JM, Wells GA, et al. Development of AMSTAR: a measurement tool to assess the methodological quality of systematic reviews. BMC Med Res Methodol 2007;7:10.

68. Higgins JPT, Altman DG. Chapter 8: Assessing risk of bias in included studies. In: Higgins JPT, Green S, eds. Cochrane Handbook for Systematic Reviews of Interventions. Chichester (UK: John Wiley \& Sons, 2008.

69. Wells BS GA, O'Connell D, Peterson J, et al. The Newcastle-Ottawa Scale (NOS) for assessing the quality of nonrandomised studies in meta-analyses. http://www.ohri.ca/programs/clinical_epidemiology/ oxford.asp

70. Reeves BC, Deeks JJ, Higgins JPT, et al. Chapter 13: Including non-randomized studies. In: Higgins JPT, Green S, eds. Cochrane handbook for systematic reviews of interventions. Chichester (UK): John Wiley \& Sons, 2008.

71. Deeks JJ, Higgins JPT, Altman DG. Chapter 9: Analysing data and undertaking meta-analyses. In: Higgins JPT, Green S, eds. Cochrane handbook for systematic reviews of interventions. Chichester (UK): John Wiley \& Sons, 2008.

72. Sterne JAC, Egger M, Moher D. Chapter 10: Addressing reporting biases. In: Higgins JPT, Green S, eds. Cochrane handbook for systematic reviews of interventions. Chichester (UK): John Wiley \& Sons, 2008

73. Paley CA, Johnson MI, Bennett MI. Acupuncture: a treatment for breakthrough pain in cancer? BMJ Support Palliat Care 2011;1:335-8.

74. Paley CA, Bennett MI, Johnson MI. Acupuncture for cancerinduced bone pain? Evid Based Complement Alternat Med 2011;2011:1-8.

75. Lu W, Rosenthal DS. Recent advances in oncology acupuncture and safety considerations in practice. Curr Treat Options Oncol 2010;11(3-4):141-6.

76. Guyatt GH, Oxman AD, Vist GE, et al. GRADE: an emerging consensus on rating quality of evidence and strength of recommendations. BMJ 2008;336:924-6.

77. Peinemann F, Labeit AM, Thielscher C, et al. Failure to address potential bias in non-randomised controlled clinical trials may cause lack of evidence on patient-reported outcomes: a method study. BMJ Open 2014;4:e004720.

78. Peinemann F, Grouven U, Bartel C, et al. Permanent interstitial lowdose-rate brachytherapy for patients with localised prostate cancer: a systematic review of randomised and nonrandomised controlled clinical trials. Eur Urol 2011;60:881-93.

79. Schmoor C, Gall C, Stampf S, et al. Correction of confounding bias in non-randomized studies by appropriate weighting. Biom J 2011;53:369-87.

80. Cox E, Martin BC, Van Staa T, et al. Good research practices for comparative effectiveness research: approaches to mitigate bias and confounding in the design of nonrandomized studies of treatment effects using secondary data sources: the international society for pharmacoeconomics and outcomes research good research practices for retrospective database analysis task force report--part ii. Value Health 2009;12:1053-61.

81. Ioannidis JP, Haidich AB, Pappa M, et al. Comparison of evidence of treatment effects in randomized and nonrandomized studies. JAMA 2001;286:821-30.

82. Chambers D, Fayter D, Paton F, et al. Use of non-randomised evidence alongside randomised trials in a systematic review of endovascular aneurysm repair: strengths and limitations. Eur $J$ Vasc Endovasc Surg 2010;39:26-34.

83. Deeks JJ, Dinnes J, D'Amico R, et al. Evaluating non-randomised intervention studies. Health Technol Assess 2003;7: 1-173.

84. Lu AP, Jia HW, Xiao C, et al. Theory of traditional Chinese medicine and therapeutic method of diseases. World $\mathrm{J}$ Gastroenterol 2004;10:1854-6

85. Lu A, Bensoussan A, Liu J, et al. Evidence-based complementary and alternative medicine. eCAM 2013;2013:723659.

86. Lu A, Xue C, Bian Z, et al. TCM Zheng classification and clinical trials 2014. Evid Based Complement Alternat Med 2015;2015:1-2.

87. Cheng CW, Kwok AO, Bian ZX, et al. The quintessence of traditional chinese medicine: syndrome and its distribution among advanced cancer patients with constipation. Evid Based Complement Alternat Med 2012;2012:1-7.

88. Song TJ, Leng HF, Zhong LL, et al. CONSORT in China: past development and future direction. Trials 2015;16:243. 
89. Bian ZX, Chang YH. Revised STRICTA as an extension of the CONSORT statement: more items should be involved in the checklist. J Altern Complement Med 2011;17:97-8.

90. Bian ZX, Shang HC. CONSORT 2010 statement: updated guidelines for reporting parallel group randomized trials. Ann Intern Med 2011;154:290-1.

91. Chen M, Cui J, Zhang AL, et al. Adherence to CONSORT items in randomized controlled trials of integrative medicine for colorectal cancer published in chinese journals. J Altern Complement Med 2017.

92. Lu L, Liao M, Zeng J, et al. Quality of reporting and its correlates among randomized controlled trials on acupuncture for cancer pain: application of the CONSORT 2010 statement and STRICTA. Expert Rev Anticancer Ther 2013;13:489-98.
93. Peng L, Min S, Zejun Z, et al. Spinal cord stimulation for cancer-related pain in adults. Cochrane Database Syst Rev 2015;6:CD009389.

94. Higgins JPT, Green S, Cochrane handbook for systematic reviews of interventions version 5.1.0. 2011. The Cochrane Collaboration. updated March 2011. http://handbook.cochrane.org

95. Paul M, Leibovici L. Systematic review or meta-analysis? Their place in the evidence hierarchy. Clin Microbiol Infect 2014;20:97-100.

96. Atkins D, Best D, Briss PA, et al. Grading quality of evidence and strength of recommendations. BMJ 2004;328:1490.

97. Petrisor B, Bhandari M. The hierarchy of evidence: levels and grades of recommendation. Indian J Orthop 2007;41:11-15.

98. Ballard M, Montgomery P. Risk of bias in overviews of reviews: a scoping review of methodological guidance and four-item checklist. Res Synth Methods 2017;8:92-108. 